\title{
Soxhlet Extractions
}

\section{Lane C. Sander}

National Institute of Standards and Technology, Gaithersburg, MD 20899, USA

lane.sander@nist.gov

Video DOI: http://doi.org/10.18434/T4DK5T

Key words: environmental analysis; extraction; quantitation; quantitative recovery; sample matrix; Soxhlet.

Accepted: December 1, 2016

Published: January 12, 2017

https://doi.org/10.6028/jres.122.004

\section{Summary}

Soxhlet extraction is one of several sample preparation methods that can be used to transfer targeted analytes from a sample matrix into a solvent, to permit subsequent analysis. With Soxhlet extraction, samples are repeatedly extracted with solvent regenerated by distillation through a periodic siphoning process. Because pure solvent is used for each extraction cycle, the removal of the analytes from the sample matrix is thermodynamically favored compared with simply soaking the sample with a single aliquot of solvent for the same period of time. This presentation will provide an introduction to the practice of soxhlet extractions, and will demonstrate the technique for a typical sample. ${ }^{1}$

\footnotetext{
${ }^{1}$ Contribution of the National Institute of Standards and Technology. Not subject to copyright. Certain commercial equipment, instruments, or materials are identified to specify adequately the experimental procedure. Such identification does not imply recommendation or endorsement by the National Institute of Standards and Technology, nor does it imply that the materials or equipment identified are the best available for the purpose.
} 